\title{
Questes
}

vestes Revue pluridisciplinaire d'études médiévales

$25 \mid 2013$

L'Habit fait-il le moine?

\section{Travestissement et transvestisme féminin à la fin du Moyen Âge}

\section{Marie de Rasse}

\section{CpenEdition}

Journals

\section{Édition électronique}

URL : http://journals.openedition.org/questes/81

DOI : 10.4000/questes. 81

ISSN : 2109-9472

\section{Éditeur}

Les Amis de Questes

\section{Édition imprimée}

Date de publication : 15 avril 2013

Pagination : 81-98

ISSN : 2102-7188

\section{Référence électronique}

Marie de Rasse, «Travestissement et transvestisme féminin à la fin du Moyen Âge », Questes [En ligne], 25 | 2013, mis en ligne le 01 janvier 2014, consulté le 19 avril 2019. URL : http:// journals.openedition.org/questes/81 ; DOI : 10.4000/questes.81 


\title{
Travestissement et transvestisme féminin à la fin du Moyen Âge
}

\author{
Marie DE RASSE \\ Université Paris-Sorbonne (Paris IV)
}

Un passage du Deutéronome mentionne l'échange de vêtements entre les individus de sexes opposés comme une pratique inconvenante et blasphématoire :

Une femme ne portera pas un costume masculin, et un homme ne mettra pas un vêtement de femme; quiconque agit ainsi est en abomination à Yahvé ton Dieu. ${ }^{1}$

En faisant de la femme le premier destinataire du message divin, le texte laisse à penser que cette faute est plus spécifiquement féminine ${ }^{2}$. Qu'en estil dans la réalité ? Quelle est la place véritable du travestissement féminin face à la morale religieuse et à l'ordre social à la fin du Moyen ' ge ?

Travaillant sur le paraître ${ }^{3}$ féminin en région parisienne à la fin du Moyen' ge, mon objectif est de définir les normes vestimentaires des femmes dans cette zone géographique entre 1350 et 1500 , à partir d'un large corpus parisien, composé aussi bien de miniatures, de chroniques,

\footnotetext{
${ }^{1}$ Deutéronome, XXII, 5, La Bible de Jérusalem. Traduction française sous la direction de l'École biblique de Jérusalem, Paris, Éditions du Cerf, 1998.

${ }^{2}$ Voir à ce sujet l'article de Nicole PELLEGRIN, "Le genre et l'habit. Figures du transvestisme féminin sous l'Ancien Régime ", dans Femmes travesties : un "mauvais" genre, Clio, 10 (1999), http://clio.revues.org/index252.html, § 5 (page consultée le 12 novembre 2011).

${ }^{3}$ Le paraître est en soi une notion, dont l'étude ne peut être abordée ici ; lors de précédents travaux (mémoire de maîtrise), j'avais défini en soulignant qu'il ne dépendait pas uniquement du vêtement, mais plutôt d'une allure, d'une apparence, et qu'il jouait un rôle important dans la définition de l'honneur de la personne. Les dimensions du présent article ne nous permettent pas d'approfondir ici la notion de " paraître ». L'on propose néanmoins de définir le "paraître » comme un ensemble composé non seulement du vêtement, mais également de l'apparence et des gestes; le paraître est en outre fortement impliqué dans la définition de l'honneur d'une personne. Le "paraître » est aussi en lien avec la notion de "représentation », et donc avec l'image publique, ici plus spécifiquement l'image féminine.
} 
d'actes de la pratique (testaments et procès), que de textes littéraires. Or, qui dit normes dit également manquements et contournements : d'après le passage du Deutéronome cité ci-dessus, travestissement et transvestisme sont à compter au nombre de ces transgressions. Le terme travestissement peut en effet revêtir deux significations : la première, " action ou manière de travestir $»^{4}$, est synonyme de déguisement ; la seconde, "déformation, parodie ", désigne une transformation mensongère, qui vise à défigurer un aspect. En revanche, le terme transvestisme, emprunté à l'anglais, s'utilise pour désigner plus particulièrement le travestissement en personne de l'autre sexe : on parle aussi de «travestissement transgenre »" L'exemple de travestissement le plus connu, et le plus débattu, est celui de Jeanne d'Arc, dont les pratiques vestimentaires ont joué un rôle lors de sa condamnation : mais s'agit-il là d'un événement isolé ou, au contraire, d'un fait révélant des pratiques relativement bien acceptées par la société de l'époque? Il faut noter que l'apparition du vêtement court pour les hommes, à la fin du XIV ${ }^{\mathrm{e}}$ siècle, accentue la différence sexuée entre les individus. Tandis que les femmes conservent une robe longue, les hommes adoptent des chausses moulantes et un manteau court - hormis pour les hommes de lois et les « sages » qui conservent le vêtement de dessus long ${ }^{6}$. Les hommes exhibent leurs jambes, les femmes les cachent: la différenciation des sexes est alors rendue visible par le vêtement, et certains habits, comme les braies ou les chausses, prennent un caractère presque symbolique.

${ }^{4}$ Le Petit Robert. Dictionnaire alphabétique et analogique de la langue française [1967], Alain ReY et Josette REY-DEbove, Paris, Dictionnaires Le Robert, 1977, article «Travestissement », p. 2012.

${ }^{5}$ Nicole Pellegrin, « Le genre et l'habit... », art. cit., § 2.

${ }^{6}$ Paris, BnF, fr. 230, fo 257, «Boccace et les luxurieux », illustration du De casibus virorum illustrium de Boccace, troisième quart du $\mathrm{XV}^{\mathrm{e}}$ siècle. Sur cette miniature, on observe bien la différence de vêtements entre hommes et femmes. Voir supra, Cahier des illustrations, Fig. 3, p. 126. 
Il est par ailleurs communément admis qu'à la fin du Moyen Age le simple travestissement était une pratique acceptée, aussi bien dans les hautes sphères sociales que chez les plus humbles: en témoignent les nombreuses traces conservées de carnavals et autres fêtes des fous ${ }^{7}$, ainsi que des événements comme le fameux Bal des Ardents survenu à la Cour en janvier $1396^{8}$. Il s'agit là de déguisements volontaires, encadrés, ritualisés, auxquels hommes et femmes participent. La place de ces dernières dans les représentations théâtrales, sujet longtemps ignoré des historiens, a été évoquée par Bernard Guenée ${ }^{9}$; ou plus récemment par Gabriella Parussa, qui s'est intéressée à la place et au rôle des femmes et des jeunes filles dans les Mystères ${ }^{10}$. Cette forme de travestissement, qu'elle relève de l'événement "extraordinaire» ou de la représentation théâtrale, ne fait pas partie de notre sujet, dans la mesure où elle s'inscrit dans le cadre de manifestations encadrées, donc soumises à des normes propres. C'est plutôt le travestissement féminin «quotidien» qui nous intéressera ici, aussi bien à travers la littérature que dans un certain nombre de cas concrets, qu'il s'agisse d'un simple déguisement «asexué » de la

\footnotetext{
${ }^{7}$ Voir Jacques HeERs, Fêtes des fous et carnavals, Paris, Fayard, 1983.

${ }^{8}$ En janvier 1396, à l'occasion du mariage d'une demoiselle d'honneur de la reine Isabeau de Bavière, le roi et quelques compagnons organisent un charivari au cours duquel ils revêtent des costumes d'hommes sauvages composés d'étoupe de lin, matière hautement inflammable. Au cours de la soirée, selon Froissart, le duc d'Orléans, frère $\mathrm{du}$ roi, approche une torche pour tenter de démasquer ceux qui se cachent sous ce déguisement. Les costumes prennent feu, et le roi ne doit son salut qu'à la duchesse de Berry qui l'enveloppe dans les plis de sa robe pour éteindre les flammes. Deux de ses compagnons décèdent de leurs brûlures.

${ }^{9}$ Dans les entrées royales, par exemple, les travestissements féminins font appel à des costumes qui relèvent plutôt de l'imaginaire mythologique : pour l'entrée d'Henri VI dans Paris en 1431 par exemple, les figures des sirènes, des Neuf Preuses, ou encore des femmes sauvages ont été convoquées. Voir Hugo ABEL, Histoire générale de France, depuis les temps les plus reculés jusqu'à nos jours, Paris, H.-L. Delloye, 5 vol., 1836-1843, t. IV, p. 150. Voir notamment Bernard GuÉNÉE et Françoise LEHOUX, Les Entrées royales françaises de 1328 à 1515, Paris, CNRS, "Sources d'histoire médiévale », 1968.

10 Je remercie Gabriella Parussa de m'avoir fait part de ses recherches en cours, auxquelles je me permets de faire référence ici.
} 
réalité, ou d'un travestissement « sexué », correspondant à une usurpation de l'habit masculin, que l'on nommera alors transvestisme. À cet égard, le passage du Deutéronome cité plus haut semble définir un ordre social tout entier soumis à l'interdit religieux ${ }^{11}$. Néanmoins, la littérature didactique et de nombreux cas concrets indiquent que l'attitude de la société du bas Moyen' ge, face au travestissement féminin, demeure relativement pragmatique et tolérante.

\section{Travestissement « asexué »}

Le travestissement simple, sans déguisement de l'identité sexuelle de la personne, relève des liens entre paraître et honneur. Le vêtement peut représenter une source de déshonneur pour la femme et pour l'homme, par exemple en cas de désordre vestimentaire ou de non respect des codes de sa classe sociale ${ }^{12}$. À l'inverse, il peut également permettre de restaurer un honneur bafoué, par le biais d'un travestissement, d'un subterfuge consistant à prendre l'habit d'un groupe social identifié et respectable, afin d'être assimilé à celui-ci : par exemple, prendre l'habit de pèlerin pour faire croire à un comportement religieux est une façon de se donner une apparence honorable. Pour la région parisienne, les deux principaux cas relevés de travestissement féminin «asexué » prennent place dans un contexte bien particulier, qui est celui de la religion.

${ }^{11}$ Le parti-pris de notre démonstration est de considérer que les préceptes bibliques constituent l'un des piliers fondamentaux de la définition des normes au Moyen ' ge.

${ }^{12}$ En témoignent les recommandations de l'auteur du Mesnagier de Paris à son épouse à la fin du XIV siècle : «Et avant que vous partiez de vostre chambre ou hostel, ayez par avant avisé que le colet de vostre chemise, vostre blanchet, ou de vostre coste ou seurcot ne saillent l'un sur l'autre; comme il est d'aucunes yvrongnes, foles ou non sachans qui ne tiennent compte de leur honneur ne de l'onnesteté de leur estat ne de leur maris, et vont les yeulx ouvers, la teste espoventablement levee comme un lion, leurs cheveux saillans hors de leurs coiffes et les coletz de leurs chemises et coctes l'un sur l'autre ; et marchent hommassement et se maintiennent laidement devant la gent sans en avoir honte » (Le Mesnagier de Paris, Georgina E. BRERETON, Janet M. FERRIER (éd.) et Karin Ueltschi (trad. et notes), Paris, LGF, «Le Livre de Poche. Lettres Gothiques », 1994, p. 42). 
Le premier exemple est tiré du Mesnagier de Paris, qui raconte l'histoire d'un mari trompé, cocu mais néanmoins homme d'honneur. Son épouse, qui a fui avec son amant, a été abandonnée par ce dernier. Le mari envoie alors les frères de sa femme la chercher, et prend soin de leur fournir un vêtement de pèlerin de Saint-Jacques et des coquilles. Pendant leur absence, il confie à son entourage et à ses voisins étonnés son bonheur de voir sa femme revenir de pèlerinage. Le vêtement de pèlerin permet de renforcer la réalité d'un mensonge et de détourner une situation infamante en associant une personne à un groupe social respectable ${ }^{13}$. Si on a peu de traces de l'utilisation d'un vêtement de pèlerin par des femmes, une illustration du Miroir historial de Vincent de Beauvais dans un manuscrit de 1463 permet de s'en faire une idée ${ }^{14}$. Hommes et femmes se fondent en une entité de couleur brune, celle du drap bon marché, non teint. Tous portent le même «manteau» ample - l'auteur du Mesnagier parle de « housse »-, long jusqu'aux genoux, sur lequel Marie-Madeleine coud une applique en forme de bourdon (le bâton spécifique du pèlerin); et tous portent le même chapeau en forme de pain de sucre. Les seuls éléments qui distinguent les hommes de la femme, qui se tient au premier plan, sont la robe qu'elle porte sous le manteau et le voile blanc qui apparaît sous le chapeau. Le manteau de pèlerin permet donc de recouvrir les habits spécifiques du sexe, ce qui, dans le cas cité par l'auteur du Mesnagier, permet d'assimiler la femme infidèle et repentie à un groupe asexué et honorable, celui des pèlerins.

Une lettre de rémission présente le cas inverse. En 1399, Perrin Larchier, un jeune clerc, demande rémission pour l'enlèvement, puis

${ }^{13}$ Le Mesnagier de Paris, éd. cit., p. 318 : « et qu'elle feust vestue de housse et chargie de coquilles a l'usage de pelerins venans de Saint Jaques ».

${ }^{14}$ Paris, BnF, fr. 50, fo 342, « Sainte Marie-Madeleine et les pèlerins », illustration du Speculum Historiale de Vincent de Beauvais, Paris, 1463. Voir supra, Cahier des illustrations, Fig. 4, p.126. 
l'abandon, de Jehannette la Flamenge, religieuse du couvent des Cordelières de Provins ${ }^{15}$. L'intérêt de cette affaire réside dans le fait que, pour voyager sans attirer l'attention, et sur les conseils de son amant, la jeune femme quitte son habit de religieuse pour prendre l'habit séculier, et revêt des habits neufs que Perrin a fait tailler pour l'occasion ${ }^{16}$. L'habit n'est malheureusement pas décrit en détail, mais la lettre mentionne «certains cuevrechiefz, anneaux et verges d'or» qu'elle emporte dans sa fuite, et dont on suppose qu'ils n'appartiennent pas à l'habit régulier. Ici, le travestissement vise à cacher son statut religieux, et à l'assimiler à un groupe « opposé », celui des laïcs, en cachant son abandon de la religion, afin qu'elle redevienne une femme semblable aux autres, et non plus une religieuse.

Ces deux exemples témoignent d'un travestissement ambivalent qui s'apparente à l'usurpation d'identité, dans la mesure où il vise à intégrer une femme à un autre groupe social que le sien, que ce soit par l'insertion dans un groupe social considéré comme respectable ou, à l'inverse, par la dissimulation d'une appartenance. Cette usurpation d'identité permet de dissimuler une faute : dans le premier cas, elle permet de se donner une respectabilité, dans le deuxième, elle cache un manque d'honorabilité. Ce type de situation semble réservé au sexe féminin : à ma connaissance, il n'existe pas d'exemple d'homme se travestissant pour sauver son honneur. Faut-il y voir une spécificité liée à la condition féminine dans l'esprit des auteurs contemporains ? Doit-on supposer que, dans l'esprit des clercs du

\footnotetext{
${ }^{15}$ L'aventure commence par un enlèvement de nuit, suivi d'un périple jusqu'au mont Saint Michel, et se termine par un retour à Provins, où l'amant abandonne la jeune femme, au couvent, alors qu'elle est enceinte. La lettre de rémission porte spécifiquement sur l'enlèvement et l'abandon de la jeune femme : le fait qu'elle ait quitté l'habit religieux n'est qu'un détail mentionné dans la narration des faits, et ne fait pas l'objet du procès.

${ }^{16}$ Choix de pièces inédites relatives au règne de Charles VI, Louis DouËT-D'ARCQ (éd.), Paris, Renouard, «Publications pour la Société de l'Histoire de France », 1844, t. II, p. 15 : « et incontinent firent vestir ladicte religieuse en habit séculier, que ils lui avoient fait faire, tout propre pour mieulx la mener par le pays ».
} 
Moyen' ge, si sensibles parfois aux traditions misogynes, les femmes, de par leur caractère de « filles d'Ève » portées à la faute, sont plus facilement susceptibles de recourir à des subterfuges -notamment pour conserver une honorabilité de façade?

\section{Transvestisme}

Cependant, la majorité des travestissements relèvent du transvestisme, c'est-à-dire de la prise de l'habit du sexe opposé. Dans la littérature et l'hagiographie, cet acte apparaît régulièrement: il peut soit témoigner d'une volonté, d'une décision réfléchie, qui répond à une situation problématique ou dangereuse, soit résulter d'une suite d'événements indépendants de la volonté.

\section{Le transvestisme volontaire}

Face à une situation particulière, la femme peut donc décider de prendre l'habit masculin. Du cas de Jeanne d'Arc, déjà largement débattu et étudié $^{17}$, je ne retiendrai ici que l'importance qu'a eue son travestissement en homme lors de la condamnation. Certes, le port du vêtement d'homme n'a pas été le premier chef d'accusation, mais il a contribué à la faire déclarer relapse, au prétexte que cette seconde transgression vestimentaire était une transgression de «la loi divine, [de] la sainte écriture, et [d]es ordonnances canoniques $»^{18}$. Toutefois, il semble que le port du vêtement masculin par les femmes ait été plus toléré qu'on ne le croit. Qu'il s'agisse de sources littéraires ou de textes plus pratiques (procès, lettres de rémission), les témoignages concernant des femmes qui prennent l'habit masculin ne manquent pas.

\footnotetext{
17 Voir Adrien HaRmand, Jeanne d'Arc, ses costumes, son armure, Paris, Leroux, 1929.

${ }^{18}$ Nicole PeLlegrin, « Le genre et l'habit... », art. cit., § 9.
} 
Dans la littérature, et notamment dans la littérature didactique destinée aux femmes, le travestissement en homme répond à des situations exceptionnelles. La Cité des Dames de Christine de Pizan peut être, par certains aspects, et notamment par son recours fréquent à des cas exemplaires, considérée comme une œuvre didactique. Cependant, elle est loin de s'adresser uniquement à un public féminin, auquel elle prétendrait prodiguer des conseils de conduite ${ }^{19}$. Au contraire, l'ouvrage se veut un panégyrique des femmes en réponse à leurs détracteurs, et s'adresse à celles-ci aussi bien qu'aux clercs et aux chevaliers. Or le transvestisme y est mentionné à plusieurs reprises comme stratagème : grâce à lui, les femmes des Lacédémoniens et sainte Nathalie peuvent prendre soin de leurs maris emprisonnés, sainte Marine peut veiller sur son père entré au couvent, et sainte Euphrosine peut protéger sa virginité lors d'un voyage ${ }^{20}$.

Un récit, dans La Cité des Dames, loue ainsi le courage des Lacédémoniennes, épouses des Minyens, qui, en se substituant à leurs maris, les font sortir de la prison où ils ont été enfermés pour cause de complot. Dans ce cas, le transvestisme devient même un acte de bravoure ${ }^{21}$.

${ }^{19}$ Ce but est plus net dans le Trésor de la Cité des Dames, ou Livre des trois vertus, qui prend la suite de La Cité des Dames, et se présente effectivement comme une suite de recommandations adressées aux femmes en fonction de leurs statuts sociaux (CHRISTINE DE PizAn, Le Livre de la Cité des Dames, Éric Hicks et Thérèse MoREAu (trad.), Paris, Stock, "Stock Moyen ' ge », 1986 ; Christine DE PIZAN, Le Livre des trois vertus, Charity Cannon WILlard et Éric Hicks (éd.), Paris/Genève, Champion, "Bibliothèque du XV $\mathrm{XV}^{\mathrm{e}}$ siècle », 1989). Si le mode de composition de cette suite montre que La Cité des Dames pouvait elle aussi être comprise comme un ouvrage didactique, la Cité ellemême se présente moins comme un recueil de conseils que comme un ouvrage écrit à la louange des dames, à l'instar du De mulieribus claris de Boccace, auquel elle emprunte nombre d'exemples.

${ }^{20}$ Christine De Pizan, Le Livre de la Cité des Dames, éd. cit., p. 160 et p. 263-266. Voir l'article de Frédérique Villemur, "Saintes et travesties du Moyen ' ge », Histoire, femmes et sociétés, Clio, 10, 1999, mis en ligne le 22 mai 2006, http://clio.revues.org/253 (page consultée le 12 novembre 2011).

21 Christine De Pizan, Le Livre de la Cité des Dames, éd. cit., p. 160 : «Elles trouvèrent enfin un stratagème : elles se vêtirent de vieux vêtements et se cachèrent la tête sous la cape de leurs manteaux, comme si elles ne voulaient pas être reconnues. Ainsi affublées, elles se rendirent à la prison. [...] Une fois en leur présence, elles les 
Une illustration montrant les Minyens sauvés par leurs épouses, dans un manuscrit du De mulieribus claris de Boccace daté de 1402, permet justement de visualiser ce travestissement: le groupe de femmes a revêtu des houppelandes et arbore des coiffes masculines, tandis que les hommes portent de simples robes et un chaperon ouvert exclusivement féminin ${ }^{22}$. Le seul élément qui permet de révéler la supercherie au lecteur est la présence d'une pilosité spécifique : les femmes ont des cheveux visiblement assez longs et leurs visages glabres s'opposent aux barbes arborées par leurs maris.

Les cas de sainte Marine et de sainte Euphrosine diffèrent un peu. Ces deux vierges prennent un vêtement masculin et religieux, Marine pour rester auprès de son père qui s'est fait moine, Euphrosine pour fuir le mariage et protéger sa virginité vouée à Dieu. Dans les deux cas, nous sommes en présence d'un double travestissement ${ }^{23}$ - prise de l'habit régulier et port de vêtements d'homme. Aucune des deux saintes ne semble rencontrer de difficulté à leurrer son entourage sur son sexe; c'est seulement à la mort de Marine que son identité sexuelle est découverte, la lavant ainsi de l'accusation d'avoir mis enceinte une femme.

Christine de Pizan ne se contente donc pas de défendre les femmes : elle réhabilite aussi les pratiques vestimentaires trans-genres, puisque le transvestisme devient une pratique relevée comme mémorable non pour le

habillèrent des vêtements qu'elles portaient, prenant pour elles ceux dont ils étaient vêtus. Alors elles les firent sortir; les gardiens crurent que c'étaient les femmes qui repartaient. ». Cette histoire présente des points communs avec celle de sainte Nathalie, épouse d'Adrien, qui prend l'habit d'homme pour pouvoir continuer à rendre visite à son mari emprisonné par l'empereur Maximilien Hercule pour s'être converti au christianisme, p. 270-271.

${ }^{22}$ Paris, BnF, fr. 12420, $\mathrm{f}^{\circ} 43 \mathrm{v}^{\circ}$, « Les Minyens sauvés par leurs épouses », illustration du De mulieribus claris de Boccace, Paris, 1402. Voir supra, Cahier des illustrations, Fig. 5, p. 126.

${ }^{23}$ Frédérique Villemur pose la question d'un «double travestissement», puisque sainte Marine n'est pas reconnue par les femmes comme telle, et qu'elle est accusée par une femme (Frédérique Villemur, « Saintes et travesties du Moyen ' ge », art. cit., en ligne). 
blâmer, comme dans le procès de Jeanne d'Arc, mais pour louer son efficacité pratique et sa sagesse. Le transvestisme, autant sinon plus qu'une transgression, devient un acte qui répond aux plus hautes vertus de chaque catégorie sociale ${ }^{24}$, et cette vertu que manifeste son emploi devient plus importante et plus notable que l'infraction à la norme. La contestation de l'infériorité féminine amène ainsi Christine à relativiser l'importance de la distinction entre hommes et femmes et, de ce fait, à ne plus prendre en compte le caractère négatif du «déguisement», qui n'est plus si violemment transgressif à partir du moment où la commune nature humaine prime sur la différence de sexe.

Le transvestisme ne se limite pas aux seules saintes de la littérature exemplaire. Dans la réalité du quotidien, le port du vêtement masculin est souvent une protection face à la promiscuité avec les hommes, comme le montrent des sources plus concrètes ${ }^{25}$.

Le cas de Jeanne d'Arc est le plus connu et le plus étudié de ces cas concrets ; c'est même « la travestie la plus populaire de la chrétienté ${ }^{26}$.

\footnotetext{
${ }^{24}$ Les Minyennes, qui le pratiquent dans un milieu laïque et chevaleresque, brillent par leur bravoure, qui est la vertu la plus prisée des chevaliers ; Euphrosine et Marine sont des saintes, et représentent donc la vertu dans les ordres religieux. Ces femmes montrent ainsi qu'elles sont capables de se faire les égales des hommes, et de pratiquer les mêmes vertus qu'eux avec autant de succès, sinon plus.

${ }^{25}$ La façon dont Euphrosine utilise le transvestisme pour protéger sa virginité peut d'ailleurs être considérée, d'un certain point de vue, comme une version hyperbolique de cette fonction. On en trouve par ailleurs plusieurs exemples dans le Décaméron de Boccace (BocCACE, Décaméron, Christian BEC (éd.), Paris, LGF, « Le Livre de Poche. Bibliothèque classique », 1994). En II-3 par exemple, la fille du roi d'Angleterre voyage travestie en moine pour se rendre à Rome ; en II-9, Zinevra, pour fuir la colère de son mari Bernardo de Gênes, se travestit en homme, et se fait embaucher comme marin, avant d'entrer au service du sultan d'Alexandrie. Il est intéressant de souligner que, dans cet exemple, la femme abandonne ses vêtements de femme, mais taille dans sa propre chemise (en la raccourcissant, donc) une paire de braies, pour prendre l'allure d'un marin. Ce fait est à la fois symbolique, puisqu'elle fabrique elle-même le vêtement porté uniquement par les hommes, les braies, qui représentent le pouvoir masculin, mais apporte aussi un élément pratique au témoignage, puisqu'elle utilise le tissu à sa disposition. De la chemise longue, symbole de la pudeur féminine, elle fait des braies, symboles de la masculinité.

${ }^{26}$ Frédérique VILLEMUR, « Saintes et travesties du Moyen ' ge », art. cit., § 37.
} 
Un seul point nous intéressera en l'occurrence. L'auteur du Journal d'un bourgeois de Paris relate, en 1431, la capture de Jeanne devant Compiègne et son procès à Rouen : dans la description qu'il donne de ses vêtements, il insiste sur le nombre d'aiguillettes qu'elle a nouées à ses chausses, et qui la rend risible aux yeux de certains $^{27}$. Ce détail permet de souligner un élément important en ce qui concerne les motivations du transvestisme féminin. En effet, l'aiguillette est l'élément constituant l'une des principales différences entre le vêtement féminin «ouvert» qui laisse le corps de la femme accessible, puisque pour y attenter il n'y a qu'un simple voile de tissu à soulever - l'historienne Nicole Pellegrin parle même de la «pénétrabilité des tenues féminines ${ }^{28}$ - et le vêtement masculin « fermé », cousu et attaché. Dans le cadre du transvestisme féminin, c'est bien souvent ce rempart de fil et de tissu qui est recherché comme protection.

C'est d'ailleurs la volonté de protection qui motive le port de l'habit masculin dans les récits de voyage. On le retrouve, toujours dans le Journal d'un Bourgeois de Paris, à l'occasion du récit de la vie de Claude des Armoises, la « fausse Jeanne », traînée en justice en 1440 pour imposture. Elle justifie la nécessité de son travestissement par les dangers d'un pèlerinage à Rome. Or le bourgeois semble davantage choqué par le fait qu'elle ait frappé sa mère que par son engagement comme soldat dans les troupes papales d'Eugène $\mathrm{IV}^{29}$. Le port volontaire du vêtement masculin n'est ainsi pas forcément condamné, surtout lorsqu'il s'agit de protéger le corps féminin.

${ }^{27}$ Le Journal d'un bourgeois de Paris : de 1405 à 1449, Colette BEAUNE (éd.), Paris, LGF, «Le Livre de Poche. Lettres Gothiques », 1990, p. 294 : « chausses vermeilles attachées à foison d'aiguillettes, dont aucuns grands seigneurs et dames lui disaient en la reprenant de la dérision de sa vêture ».

${ }^{28}$ Nicole PELLEGRIN, « Le genre et l'habit... », art. cit., $\S 21$.

29 Le Journal d'un bourgeois de Paris, op. cit., p. 398 : «Et pour cette cause lui convenait aller a Rome ; et pour ce elle y alla vêtue comme un homme et fut comme soudoyer en la guerre du Saint Père Eugène ». 


\section{Le transvestisme involontaire}

Dans certains cas, la prise de l'habit masculin peut aussi s'avérer involontaire, bien que nécessaire, notamment en situation de grande pauvreté, ou lorsque des femmes héritent de vêtements.

Une lettre de Charles VI, datée de 1400, accorde rémission à Colin Petit, pour avoir abusé, lors d'un passage en région parisienne, d'une femme muette, que le mari de celle-ci lui avait présentée comme une prostituée réputée des environs de Chartres. Vraisemblablement pris de pitié, et trouvant sa victime trop mal habillée, l'agresseur la ramène à son époux vêtue de sa propre houppelande et d'un chaperon ouvragé, lui faisant don de sa ceinture et d'une dague en prime ${ }^{30}$. Dans ce cas, on peut dire que la jeune femme porte des habits d'homme par nécessité.

La situation des femmes qui héritent de vêtements masculins est plus complexe à analyser. L'étude de quinze testaments d'hommes, pour une période comprise entre 1392 et 1421, révèle que dans 40,5\% des actes, les testateurs lèguent une partie de leurs vêtements à des femmes. Il s'agit essentiellement de robes (39\% des dons), de chaperons $(16 \%)$ et de houppelandes (10\%), c'est-à-dire de vêtements d'extérieur, qui sont aussi les moins nettement sexués. Le reste se répartit entre diverses pièces :

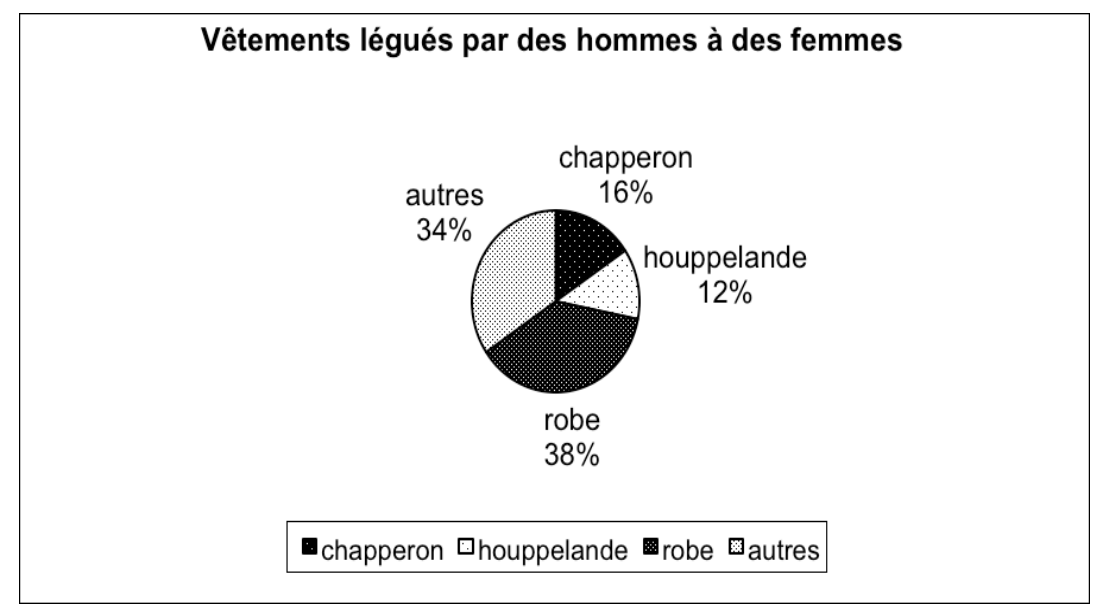

${ }^{30}$ Choix de pièces inédites relatives au règne de Charles VI, op. cit., t. II, p. 245 : " vestue d'une houppelande à homme, d'une sainture et d'une dague, et d'un chapperon décoppé $[. .$.$] pour ce qu'elle estoit très mal vestue » (nous soulignons).$ 
Mais si nous savons à qui ces vêtements sont légués, et même si certains testateurs stipulent qu'ils sont destinés à l'une ou l'autre "pour elle vestir $»^{31}$, nous n'avons aucune indication sur le fait que les héritières les aient effectivement portés, tels quels ou retouchés. Il est aussi possible que cet héritage ait été revendu pour utiliser l'argent, car les vêtements représentent de réels biens de valeur, et les vêtements légués sont de plus en draps de bonne qualité. En effet, la majorité est d'écarlate, c'est-à-dire de drap de laine fin ; mais il est aussi fait mention de drap d'Angleterre, de soie, et, même de racami, c'est-à-dire d'un drap de soie broché d'or ou d'argent. L'étude de l'identité des héritières citées par ces testaments permet de dégager un schéma de trois cercles concentriques, allant de la famille, proche ou éloignée, puis de la domesticité, aux dons de charité. Il est intéressant de souligner que certains testateurs donnent priorité à leurs domestiques, chambrières ou servantes, ou bien leur destinent des vêtements coûteux, comme c'est le cas pour Margarete, servante de Jean d'Essoyes, qui hérite en 1403 d'une houppelande longue de drap violet fourrée de gris $^{32}$, ou pour Philippote, la servante du notaire Aimeri de Montragoux, qui hérite d'une cotte de racami en $1405^{33}$. Cette inversion dans la hiérarchie classique des dons peut s'expliquer par la volonté de remercier un domestique fidèle, parfois plus proche de son maître que la propre famille de celui-ci. L'étude du testament de Jean de Neuilly-Saint-Font, établi en 1402, laisse à penser que ces vêtements sont effectivement destinés à être portés, puisque les cottes longues sont

\footnotetext{
${ }^{31}$ Testaments enregistrés au Parlement de Paris sous le règne de Charles VI, Alexandre Tuetey (éd.), Paris, Imprimerie Nationale, 1880, p. 326, testament de Jean Noyers, janvier 1415: "à la sœur de feu maistre Jaques Loron, pour elle vestir" (nous soulignons).

32 Ibid., p. 112 : «item, legavit Margarete, ancille sue [...], unam longuam hoppelandam de violeto, foderatam de grisiis $»$.

33 Ibid., p. 169 : «Item, il laissa a Philippote qui l'avoit servi sa cote de racami ».
} 
léguées aux femmes et les habits courts aux hommes ${ }^{34}$. Cela sous entend qu'il suffit de peu de retouches pour faire correspondre la silhouette au sexe de son porteur.

Ainsi, l'ensemble de ces exemples montre que, dans certaines occasions, des femmes peuvent se trouver en possession de vêtements d'hommes, et les porter, sans pour autant représenter une menace pour l'ordre social établi. L'adoption du vêtement masculin déféminise celle qui le porte, mais ne prête pas à conséquences, aussi longtemps qu'il n'a pas pour but d'usurper le rôle ou le pouvoir masculin. Le travestissement féminin existe et est toléré dans la société du bas Moyen' ge aussi longtemps que la répartition des rôles est respectée. Notons que, pour que le transvestisme féminin soit jugé acceptable, il est très important qu'il soit occasionnel et limité dans le temps. Pour cette raison, Froissart ne peut condamner l'attitude de Jeanne de Flandre, comtesse de Monfort, lors du siège d'Hennebont: en l'absence de son mari, commandant militaire de la place, elle adopte, par nécessité, l'habit et le «harnois » masculin pour mener ses gens au combat, mais reprend l'habit normal de son «estat» aussitôt le danger passé ${ }^{35}$. Le transvestisme a là encore une visée protectrice: le but n'est plus de protéger la vertu, mais simplement de protéger le corps.

\section{Le transvestisme masculin}

Par opposition, dans les représentations qui en sont données, le transvestisme masculin tend généralement à la luxure, et non à la

\footnotetext{
${ }^{34}$ Ibid., p. 79 : « et que mes cottes longues soient distribuées aux femmes de mon lignage [...], et mes cours habiz soient distribuez entre mes autres serviteurs ».

35 JeAn Froissart, Euvres de Froissart, publiées avec les variantes des divers manuscrits, Chroniques, t. IV, 1342-1346 (depuis le siège de Rennes jusqu'à l'expédition d'Édouard III en Normandie), Joseph Bruno Marie Constantin KERVYN DE LetTENHOVE (éd.), Osnabrück, Biblio Verlag, 1967, p. 17 (réimpression de l'édition de Bruxelles, parue chez V. Devaux \& cie, en 1867-1877).
} 
protection. Cette situation, qui porte facilement à rire dans la littérature, est dans la réalité très mal tolérée par la société, qui y voit une confusion des rôles. L'homme qui se travestit en femme ne se contente pas de se déviriliser, il se féminise, renvoyant ainsi aux craintes de désordre social que suscitent les pratiques sodomites: l'homme qui abandonne ses attributions masculines s'expose à une condamnation et une répression bien plus féroces que la femme qui se les approprie.

Les témoignages sur le transvestisme masculin sont peu nombreux, mais il en existe quelques exemples dans la littérature, et plus particulièrement dans les fabliaux, où le travestissement de l'homme en femme est récurrent et produit des effets comiques ${ }^{36}$. Dans Trubert par exemple, un fabliau du XIII ${ }^{\mathrm{e}}$ siècle, le héros se fait successivement passer pour différents personnages, du charpentier au médecin, du médecin au chevalier, pour terminer par la femme, qui représente clairement, dans cette gradation, le déguisement le plus transgressif, et permet entre autres au personnage principal d'approcher une femme qu'il veut séduire ${ }^{37}$. Dans le Décaméron, Egano, mari trompé, revêt le surcot et le voile de sa femme pour tenter de confondre l'amant de celle- $\mathrm{ci}^{38}$; dans les Cent nouvelles nouvelles, un jeune écossais habitant à Rome prend des habits de femme et devient lavandière, ce qui lui permet de coucher avec un grand nombre de femmes à l'insu de leurs parents, maris ou maîtres ${ }^{39}$. Dans la fiction, la

\footnotetext{
${ }^{36}$ Soulignons tout de même que dans la mesure où la perception du transvestisme masculin est fondée sur les fabliaux, elle est forcément biaisée : ceux-ci présentent notoirement un goût prononcé pour la grivoiserie. Dans ce cadre, l'association entre transvestisme masculin et luxure est particulièrement efficace.

37 «Desvest toute ta robe tost / Si vest ma robe et mon seurquot » (DOUIN DE LAVESNE, Trubert, dans Fabliaux érotiques, Luciano Rossi (éd.), Paris, LGF, " Le Livre de Poche. Lettres gothiques », 1992, p. 345-529, v. 2267-2269).

${ }^{38}$ BocCaCE, Décaméron, éd. cit., $7^{\mathrm{e}}$ journée, $7^{\mathrm{e}}$ nouvelle, p. 571.

39 Les Cent Nouvelles Nouvelles, Pierre CHAMPION (éd.), Paris, Droz, « Documents

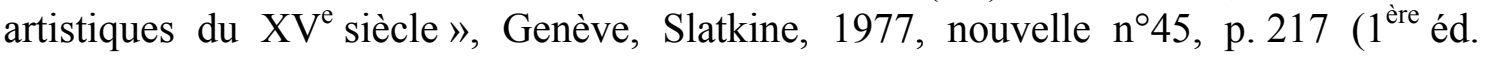
1928).
} 
dévirilisation de l'homme a pour enjeu l'approche d'une femme, elle prête donc à rire ${ }^{40}$.

Dans la réalité également, le transvestisme masculin ne semble avoir d'autre but que la sexualité, et sert généralement un type de prostitution jugé particulièrement immoral. $\mathrm{Si}$, à ma connaissance, aucun texte législatif laïque n'interdit le travestissement en femme, la morale religieuse, en revanche, le désapprouve fortement, et l'interdiction du transvestisme fait l'objet d'une législation ecclésiastique ${ }^{41}$. Dans les faits, et hors de certaines situations «encadrées 》 (comme la fête de l'âne ou les rites carnavalesques), la prise de l'habit féminin mène bien souvent à l'accusation de sodomie. En témoignent des procès, comme celui, tenu à Reims en 1372, d'un dénommé Raymond, jugé et condamné pour travestissement en femme et prostitution à la suite d'une plainte déposée par un client furieux de s'être laissé trompé par l'apparence ${ }^{42}$. Contrairement au transvestisme féminin, qui se veut protecteur et peut donc être accepté dans certaines circonstances en dépit de l'interdiction proférée par le Deutéronome, le transvestisme masculin est donc frappé par un interdit à la fois religieux et social, car son but est essentiellement la luxure.

\footnotetext{
${ }^{40}$ Je tiens cependant à souligner un point relevé par Fanny Oudin lors du séminaire : dans ces exemples, mais plus particulièrement dans le cas de Trubert, la portée de ce rire et ses rapports à la morale sont ambigus, car le fabliau est un genre qui repose sur le mélange de la vérité et du doute, et se joue des attentes de son lecteur. Dans les recueils de nouvelles, qui héritent certains de leurs procédés des fabliaux, la morale est plus souvent exprimée de manière explicite, mais elle est brouillée par le débat entre les auditeurs du conte. Dans ces conditions, la portée du rire que fait naître le récit reste toujours ambiguë, d'autant plus qu'il est fréquemment lié au plaisir de la transgression temporaire de tabous, et peut en ce sens avoir éventuellement une dimension cathartique (sur cette question, voir Dominique Boutet, Les Fabliaux, Paris, PUF, «Études littéraires », 1985). Cette tension entre le didactisme et le jeu est d'ailleurs en accord avec le goût de ces récits pour la manipulation, dont le déguisement est justement une forme.

${ }^{41}$ Voir Frédérique Villemur, « Saintes et travesties du Moyen ' ge », art. cit., en ligne, $\S 1$ : concile de Gangres 431, Code Théodosien 435.

42 Florent Véniel, La Sexualité au Moyen Âge, Aubagne, la Muse, "Moyen ' ge », 2008, p. 93.
} 
Au terme de ce parcours, il semble donc que l'idée communément admise d'un bas Moyen ' ge très hostile au transvestisme féminin doive être écartée. Même si les interdits religieux sont souvent évoqués comme base légale de condamnations, il s'avère que dans les faits la société de l'époque a une approche beaucoup plus souple et pragmatique. En apparence, l'interdit religieux semble toujours primer, mais, dans la pratique, les règles d'ordre social, tacites, sont essentiellement liées à la peur des autorités qui craignent la subversion politique et morale. Cette crainte $^{43}$, caractéristique du Moyen ' ge, perdure au long des siècles, et l'interdit religieux finit par devenir secondaire dans les discours.

C'est donc la peur du désordre social qui conditionne le regard que portent les individus de la fin du Moyen ' ge sur le transvestisme. Dans de nombreux cas, la société tolère certains agissements que l'Église réprouve, tant qu'il ne s'agit pas d'usurper le rôle de l'autre sexe. La femme peut se « transvestir », pour faire face à des situations exceptionnelles, mais doit revenir à son «estat» sitôt le problème résolu, sous peine de menacer l'ordre naturel des choses. Aucun des attributs vestimentaires masculins ne lui est interdit à l'exception du seul qui incarne le rôle et la place de l'homme : les braies, c'est-à-dire les sous-vêtements.

L'approche de la société médiévale est donc duelle : tolérante face à des circonstances atypiques, elle se révèle inflexible sur la « bataille pour la culotte ${ }^{44}$. Cette bataille n'est qu'une des nombreuses facettes du

${ }^{43}$ Cette crainte est perceptible dans le Journal d'un Bourgeois de Paris, à l'année 1446 : l'auteur écrit en effet que la fin des temps viendra lorsque «tous jeunes gens seront deguisés d'habit, tant femmes que hommes » (Journal d'un Bourgeois de Paris, éd. cit., p. 431).

44 Pierre BUREAU, « La "dispute pour la culotte". Variations littéraires et iconographiques d'un thème profane (XIII $-\mathrm{XVI}^{\mathrm{e}}$ siècles) », dans L'Étoffe et le vêtement, Médiévales, 29, 1995, p. 105-129. 
transvestisme médiéval, mais a gagné en importance au cours des siècles, et reste d'actualité ${ }^{45}$.

${ }^{45}$ À titre d'information, l'ordonnance du 16 brumaire an IX, interdisant aux femmes de s'habiller en homme sans l'autorisation du préfet de police, n'a été abrogée qu'en janvier 2013. 